Article

\title{
The Disturbance of Hepatic and Serous Lipids in Aristolochic Acid I Induced Rats for Hepatotoxicity Using Lipidomics Approach
}

\author{
Junyi Zhou, Yifei Yang, Hongjie Wang, Baolin Bian, Jian Yang, Xiaolu Wei, Yanyan Zhou, Nan Si * \\ and Haiyu Zhao * \\ Institute of Chinese Materia Medica, China Academy of Chinese Medical Sciences, Beijing 100700, China; \\ zzjjyyy@163.com (J.Z.); yangyifei1987@163.com (Y.Y.); hjwang@icmm.ac.cn (H.W.); blbian@icmm.ac.cn (B.B.); \\ jyang@icmm.ac.cn (J.Y.); xlwei@icmm.ac.cn (X.W.); yyzhou@icmm.ac.cn (Y.Z.) \\ * Correspondence: nsi@icmm.ac.cn (N.S.); hyzhao@icmm.ac.cn (H.Z.); Tel.: +86-189-111-091-31
}

(N.S.); +86-136-610-784-32 (H.Z.)

Received: 8 September 2019; Accepted: 14 October 2019; Published: 17 October 2019

\begin{abstract}
Aristolochic acid I (AAI) was regarded as the major toxic component of aristolochic acid (AA). In addition to aristolochic acid nephropathy (AAN), liver cancers induced by AAI has aroused increasing attention recently. In this paper, the discovery of diagnostic biomarkers for AAI-induced liver injury has been studied, especially for the lipid markers. From the histopathological characteristics, the injury was observed clearly in the liver apart from the kidney after $30 \mathrm{mg} / \mathrm{kg}$ of AAI treatment for one week, while the lesion alleviated after AAI discontinuance. The serum biochemical indexes were manifested to the normal tendency after AAI discontinuance for two weeks. According to the evaluation of pathology slices and serum biochemical indexes, they indicated that the hepatotoxicity induced by AAI was reversible to some extent. A total of 44 lipid markers were identified in the liver, as well as 59 in the serum. Twenty-six common lipid markers were observed in both serum and liver. Furthermore, nine out of 26 lipids exhibited the excellent diagnostic ability to differentiate the control group from the AAI group and AAI discontinuance group with high sensitivity and specificity. The changed lipid markers might serve as characteristics to explain the mechanisms of pathogenesis and progression in hepatotoxicity induced by AAI.
\end{abstract}

Keywords: aristolochic acid I; hepatotoxicity; lipidomics; biomarkers

\section{Introduction}

Aristolochic acids (AA), a kind of nitrophenanthrene carboxylic acid compounds, were widely distributed in Aristolochia and Asarum plants of the Aristolochiaceae family. Aristolochia was extensively possessed with multi activities for antitumor, antibacterial, anti-inflammatory, analgesic biological activities, and other pharmacological effects. Meanwhile, the increasing emphasis on the toxicity of AA has been laid in recent years. Aristolochic acids I (AAI) was regarded as the major toxic chemical from Aristolochia. Aristololactam I (ALI) was the main metabolite of AAI by nitro-reduction reaction in vivo. Both the AAI and ALI could induce the apoptosis of cultured renal epithelial cells through the activation of caspase 3-dependent pathway [1].

AA could induce the apoptosis of proximal tubular cell through various mechanisms, including mitochondrial DNA depletion, respiratory chain defects, and endoplasmic reticulum stress [2,3]. Meanwhile, AA induced the accumulation of methylglyoxal (MGO) and $\mathrm{N}^{\varepsilon}$-(carboxymethyl) lysine (CML) has become an important and novel pathway in the pathogenic mechanism for aristolochic acid nephropathy (AAN). The serious kidney damage induced by AA was related to an increase and accumulation of MGO and CML. It caused the tubular atrophy with decreased renal 
function. Meanwhile, creatinine clearance, GSH levels, and the intra-renal antioxidant capacity were descended [4]. Recently, according to the reports [5,6], apart from AAN, AAI and AAI-DNA adduct could induce the liver cancer through characteristic adenine-to-thymine transversions. The results suggested that the herbal medicines containing AA had the potential mutagenic effects in liver. AAI has been listed as one of the major risk factors for liver cancers. Although these results were still controversial, the liver injury caused by AAI was obvious, which attracted increasing attention world-wide. As the continuation of this work, the discovery for the diagnostic biomarkers of AAI-induced liver injury has been considered to be very significant and urgent, especially for lipid markers.

However, in recent years, lipidomics has become a powerful tool for the safety evaluation [7]. It was defined as the completely quantitative determination of lipids in cells, tissues, or biological fluids [8,9]. The researches of lipid homeostasis disorder in liver injury induced by AAI have not been reported. Liver was a major organ for fatty acid oxidation and ketone body formation. The characteristically pathologic changes in the liver were always companied with the disturbance of lipid homeostasis. It has been reported that liver damage could notably affect the normal processes of lipid metabolism through increasing lipid synthesis, reducing the ability of fatty acid $\beta$-oxidation, inducing oxidative stress and disturbing thyroid hormone pathways in liver [10]. Thus, the systemic analysis of the disorder in lipid metabolism was a feasible approach for the diagnosis of AAI liver injury.

In the present study, the lipid biomarkers in the AAI treatment group and AAI discontinuance groups were compared to the difference among them. The histopathology of liver and kidney and the biochemical function were evaluated. The injury was identified in liver after the AAI treatment for one week, while the lesion was alleviated after AAI discontinuance for two weeks. The liver exhibited the strong tendency for self-repairing capability. To confirm the potential lipid biomarkers which could be applied in clinical diagnosis, the common lipids in both liver and serum were screened and identified. The disturbances in lipid homeostasis were closely related to the pathogenesis and progression of liver injury.

\section{Results and Discussion}

\subsection{Tissue Histological Characteristics}

The H\&E staining of liver and kidney sections were carried out (Figure 1). The histological findings indicated that in the AAI group, the liver tissue showed diffuse swelling accompanied with vacuolar degeneration in hepatocyte. In kidney, the renal tubular epithelial cells in cortex showed the multiple focal swollen. Meanwhile, the degeneration of renal tubular epithelial cells was also observed. The acidophil appeared to increase in the cytoplasm of kidney. In general, the severity of the lesion in liver was milder than that in kidney obviously. The kidney was the main impairment target organ in the AAI-induced injury. In the discontinuance groups, the mild focal hepatocyte swelling and vacuolar degeneration were observed in liver but with no significant difference. The degree of pathological changes was lightened compared to the AAI group. In kidney, multiple focal swelling, degeneration, lumen narrowing, and mesenchymal cell hyperplasia were observed. The lesion was alleviated during the kidney repair process.

\subsection{Serum Biochemical Index Results}

A total of 11 serum biochemical indexes related to the function of liver and kidney were detected, including ALT, AST, TBA, ALB, GLB, LDH, CHE, TP, Crea, BUN, and UA. Compared with the control group, the concentration of ALB, TP, and CHE were significantly decreased in the AAI group. Discontinuance of AAI for two weeks later, ALT, AST, TP, TBA, ALB, GLB, LDH, and CHE were exhibited the tendency to the normal level (Table 1). 

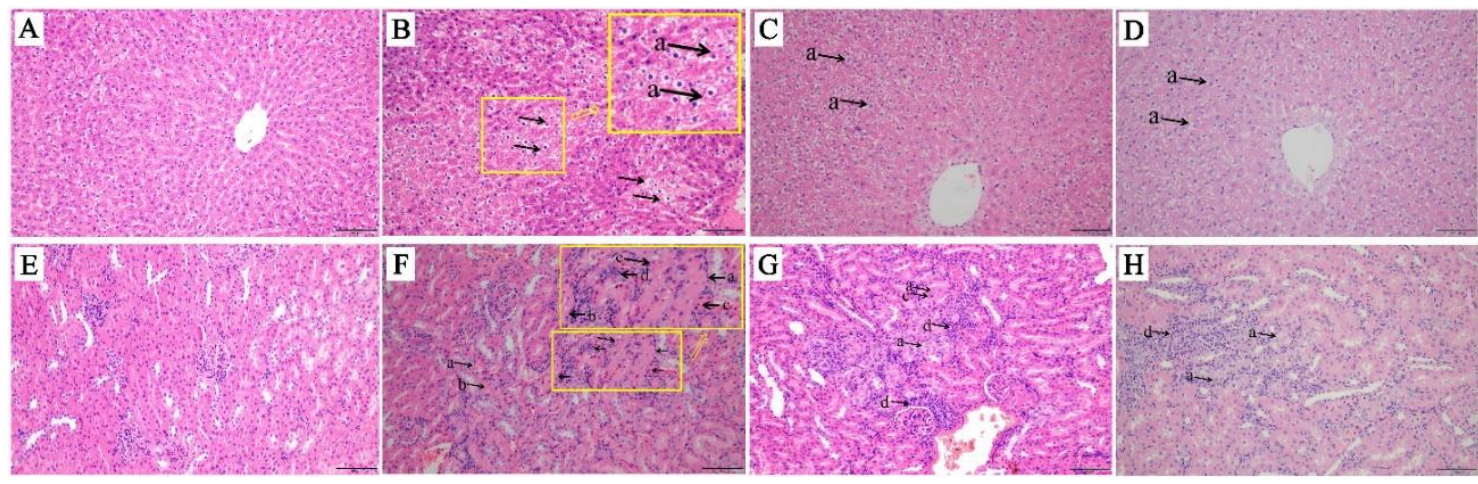

Figure 1. Liver histological structure of rats with $30 \mathrm{mg} / \mathrm{kg} / \mathrm{d}$ of aristolochic acid I (AAI) oral administration for one week and AAI discontinuance for one week and two weeks. (A) No obviously histopathological changes in the liver of the control group. (B) Diffuse swollen (in the yellow box) and vacuolar degeneration in hepatocyte $(a \rightarrow)$ in the liver of AAI group. $(\mathbf{C})$ and $(\mathbf{D})$ Mild focal hepatocyte swollen and vacuolar degeneration $(\mathrm{a} \rightarrow$ ) in the liver of one-week group and two-week group, respectively. (E) No obviously histopathological changes in the kidney of control group. (F) The epithelial cell of renal tubule in cortex showed multiple focal swollen and degeneration $(\mathrm{a} \rightarrow)$. The acidophily increased in cytoplasm $(\mathrm{b} \rightarrow)$. Necrosis, karyopyknosis, karyolysis, and even disappearance $(c \rightarrow)$ in individual epithelial cell. Hyperplasia of the mesenchymal cell around the injury renal tubules $(\mathrm{d} \rightarrow)$. $(\mathbf{G})$ and $(\mathbf{H})$ Multiple focal swollen, degeneration, lumen narrowing, mesenchymal cell hyperplasia in the kidney of one-week group and two-week group, respectively.

Table 1. Effect of AAI on serum biochemical indexes in four groups.

\begin{tabular}{|c|c|c|c|c|c|c|}
\hline Group & ALT (U/L) & AST (U/L) & TBA $(\mu \mathrm{mol} / \mathrm{L})$ & $\operatorname{ALB}(\mathrm{g} / \mathrm{L})$ & GLB (g/L) & CHE (U/L) \\
\hline Control & $52 \pm 6.07$ & $93.25 \pm 25.47$ & $28.22 \pm 17.41$ & $31.53 \pm 0.89$ & $20.60 \pm 0.48$ & $102.75 \pm 19.48$ \\
\hline AA & $64.14 \pm 24.39$ & $112.2 \pm 34.19$ & $30.16 \pm 12.99$ & $29.63 \pm 0.69$ \#\#\# & $19.87 \pm 1.76$ & $84.44 \pm 17.30^{\#}$ \\
\hline 1 week & $100.63 \pm 7.58^{* * *}$ & $132.88 \pm 27.05$ & $35.77 \pm 18.06$ & $30.29 \pm 1.27$ & $18.82 \pm 1.15$ & $107.10 \pm 17.80^{* *}$ \\
\hline 2 week & $55.40 \pm 7.04$ & $107.22 \pm 14.13$ & $20.98 \pm 11.88$ & $31.71 \pm 0.66^{* * *}$ & $19.45 \pm 0.90$ & $117.00 \pm 15.40^{* * *}$ \\
\hline Group & $\mathrm{TP}(\mathrm{g} / \mathrm{L})$ & LDH (U/L) & Crea $(\mu \mathrm{mol} / \mathrm{L})$ & BUN (mmol/L) & $\begin{array}{c}\text { UA } \\
(\mu \mathrm{mol} / \mathrm{L})\end{array}$ & \\
\hline Control & $52.43 \pm 1.71$ & $367.71 \pm 127.57$ & $15.78 \pm 1.72$ & $4.82 \pm 0.45$ & $27.63 \pm 8.28$ & \\
\hline AA & $48.54 \pm 1.42$ \#\#\# & $643.00 \pm 315.26^{\# \#}$ & $19.80 \pm 6.03^{\#}$ & $5.19 \pm 0.94$ & $28.17 \pm 19.16$ & \\
\hline 1 week & $49.05 \pm 1.60$ & $349.89 \pm 133.01 * *$ & $21.50 \pm 6.09$ & $5.37 \pm 0.63$ & $15.89 \pm 7.72$ & \\
\hline 2 week & $51.46 \pm 1.82 * * *$ & $305.67 \pm 96.07 * *$ & $22.33 \pm 1.75$ & $5.54 \pm 0.78$ & $14.00 \pm 4.12 *$ & \\
\hline
\end{tabular}

Compared with control group: ${ }^{\#} P<0.05,{ }^{\# \#} P<0.01,{ }^{\# \#} P<0.001$. Compared with AA-oral administration group: ${ }^{*} P<0.05,{ }^{* *} P<0.01, * * * P<0.001$.

AST and ALT, the liver function enzymes, could commonly assess the severity of hepatic disease [11]. Compared to the AAI group, ALT displayed a more than twofold increase in the one-week group. The concentration of ALT almost returned to the normal level in the subsequent two-week group. In this study, AST/ALT ratio was higher than 1.2 in the AAI group. Specifically, the ratio of AST/ALT $>1$ was a significant predictor of liver dysfunction [12,13]. CHE was obviously declined in AAI group compared to the control group. Due to the concentration of $\mathrm{CHE}$ in serum decrease for the liver presented symptoms of damage, CHE could not be timely supplemented in serum [14]. While compared to the AAI group, CHE remarkably increased $30 \%$ and $40 \%$ in the discontinuance groups, respectively. Meanwhile, compared to the control group, LDH and Crea were significantly increased in the AAI group, which suggested the impairment of the function of glomerular filtration.

The biochemical test items could effectively reflect the anabolic status and the degree of hepatocyte damage, so as to provide the referenced basis for the treatment and prognosis of patients with liver diseases [15]. The data showed that the disturbance in the biochemical indexes in different levels was closely related to the liver function. Interestingly, in the discontinuance groups, the majority of the aforementioned indexes presented the trend to the normal level. The liver exhibited the strong tendency of self-repairing capability. 


\subsection{Examination of Concentration of AAI and ALI in Liver and Serum}

The precursor and product ions of multiple reaction monitoring (MRM) detection were listed (Table 2). The limit of quantitation (LOQ) for AAI and ALI was $5 \mathrm{ng} / \mathrm{mL}$ based on $\mathrm{S} / \mathrm{N} \geq 10$. The method offered a good linearity. The $r$ value was above 0.99 (Tables 3 and 4). The concentrations of AAI and ALI in liver and serum in the AAI group were shown in Table 5, except for the concentration of ALI in the serum since it was below the LOQ. In addition, the concentrations of AAI and ALI in liver and serum were undetectable in the discontinuance groups.

Table 2. Optimized multiple reaction monitoring (MRM) pairs and parameters for AAI and aristololactam I (ALI).

\begin{tabular}{|c|c|c|c|c|}
\hline \multirow{2}{*}{ Analyte } & \multicolumn{2}{|c|}{$m / z$} & \multirow{2}{*}{$\mathrm{DP}(\mathrm{V})$} & \multirow{2}{*}{ CE (V } \\
\hline & Precursor & Product & & \\
\hline AAI & 359.1 & 298.8 & 66 & 15 \\
\hline ALI & 294.1 & 279.2 & 146 & 39 \\
\hline Buspirone (IS) & 386.2 & 122.2 & 91 & 49 \\
\hline
\end{tabular}

Table 3. Linearity, limit of quantitation (LOQ) of the LC-MS/MS assay for AAI and ALI in serum.

\begin{tabular}{ccccc}
\hline Analyte & Equation & Correlation Coefficient $(\mathbf{r})$ & Linear Range $(\mathbf{n g} / \mathbf{m L})$ & LOQ $(\mathbf{n g} / \mathbf{m L})$ \\
\hline AAI & $\mathrm{y}=0.000512 x+0.000337$ & 0.9954 & $5-1000$ & 5 \\
ALI & $\mathrm{y}=0.000425 \mathrm{x}-0.000135$ & 0.9928 & $5-1000$ & 5 \\
\hline
\end{tabular}

Table 4. Linearity, LOQ of the LC-MS/MS assay for AAI and ALI in liver.

\begin{tabular}{ccccc}
\hline Analyte & Equation & Correlation Coefficient $(\mathbf{r})$ & Linear Range $(\mathbf{n g} / \mathbf{m L})$ & LOQ $(\mathbf{n g} / \mathbf{m L})$ \\
\hline AAI & $\mathrm{y}=0.000403 x+0.000775$ & 0.9993 & $5-1000$ & 5 \\
ALI & $\mathrm{y}=0.00045 \mathrm{x}+0.000265$ & 0.9937 & $5-1000$ & 5 \\
\hline
\end{tabular}

Table 5. The level of AAI and ALI (ng/mL) in serum and liver in the AA group $(n=6)$.

\begin{tabular}{cccccccc}
\hline Analyte & Serum & Serum & Serum & Serum & Serum & Serum & Mean \pm SEM \\
\hline AAI & 75.1 & 67.6 & 65.5 & 81.5 & 55.5 & 52.4 & $66.27 \pm 4.55$ \\
ALI & - & - & - & - & - & - & - \\
\hline Analyte & Liver & Liver & Liver & Liver & Liver & Liver & Mean \pm SEM \\
\hline AAI & 98.6 & 50.2 & 97.0 & 95.8 & 62.5 & 79.4 & $80.58 \pm 8.32$ \\
ALI & 42.7 & 61.2 & 32.5 & 37.4 & 86.4 & 68.3 & $54.75 \pm 8.50$ \\
\hline \multicolumn{7}{r}{-: the concentration of the analyte was below the LOQ. }
\end{tabular}

\subsection{Identification of the Potential Lipid Biomarkers}

From the original MS data obtained from liver and serum samples, the multivariate statistics, unsupervised model of principal components analysis (PCA), and supervised model of orthogonal projections to latent structures discriminant analysis (OPLS-DA) were applied to gain an overview of the lipidomic profilings in all groups and set an obvious separation of the control group and AAI group respectively, with high statistical values of $R^{2}$ and $Q^{2}$. In the PCA score, the cumulative $R^{2} X$ and $\mathrm{Q}^{2}$ were $0.802,0.651$ in serum, 0.865 , and 0.727 in liver. In the OPLS-DA model, $R^{2} X, R^{2} Y$, and $Q^{2}$ were $0.865,0.976$, and 0.886 in serum, $0.725,0.936$, and 0.777 in liver. No over-fitting was observed according to the permutation (Figure 2).

The comparisons of difference among the three groups (control group, AAI group, one-week group) in liver and serum were then performed based on the parameters. In the AAI group, a total of 59 lipid biomarkers including 36 phosphatidylcholines (PCs), 13 sphingolipids (SMs), five phosphatidylethanolamines (PEs), four glucosylceramides (GlcCers), and one ceramide (Cer) were 
significantly altered in serum. Fourty-four lipid biomarkers including 22 PCs, 10 PEs, 7 SMs, 3 Cers, and 2 GlcCers were remarkably identified in liver.
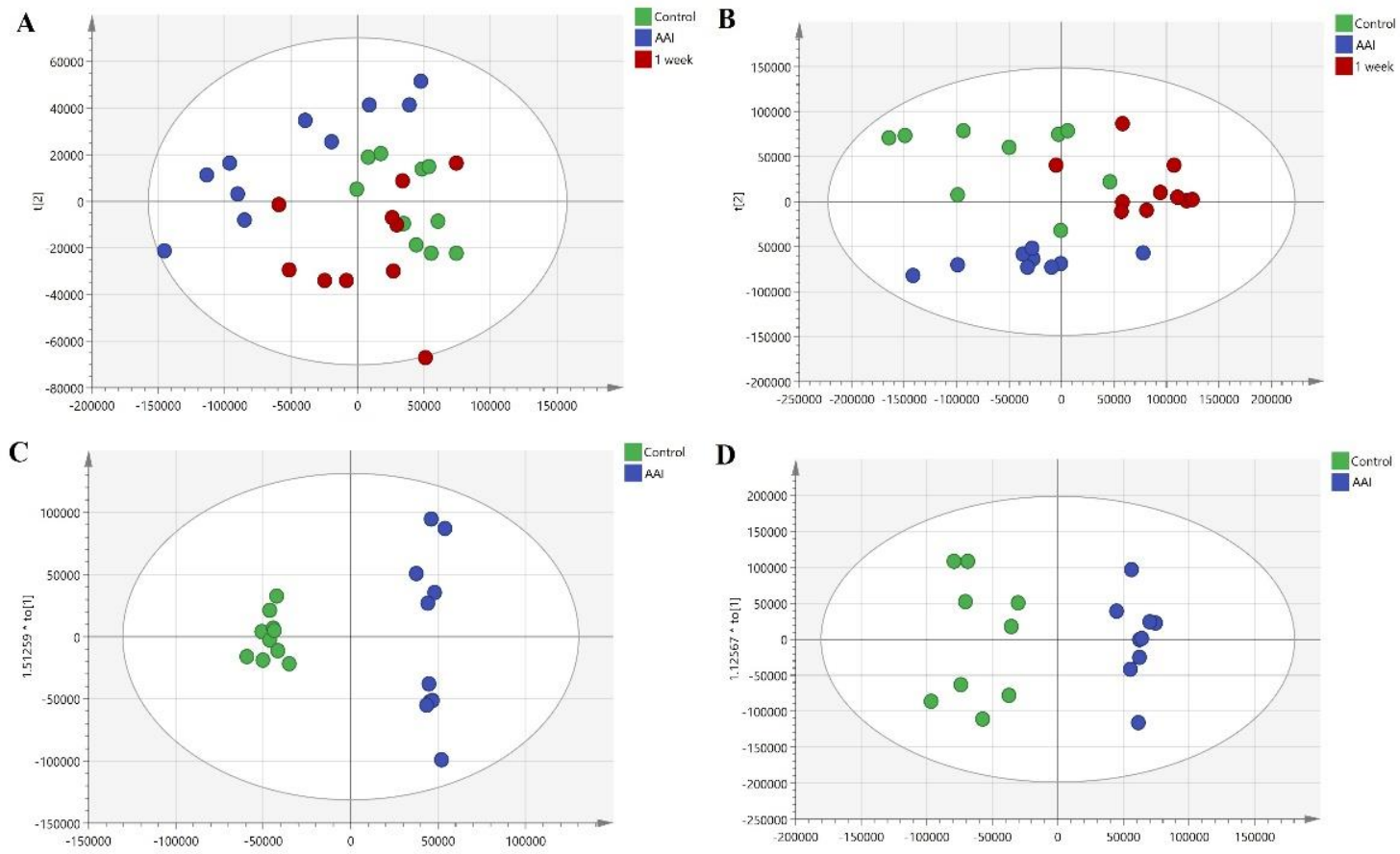

$\mathbf{E}$
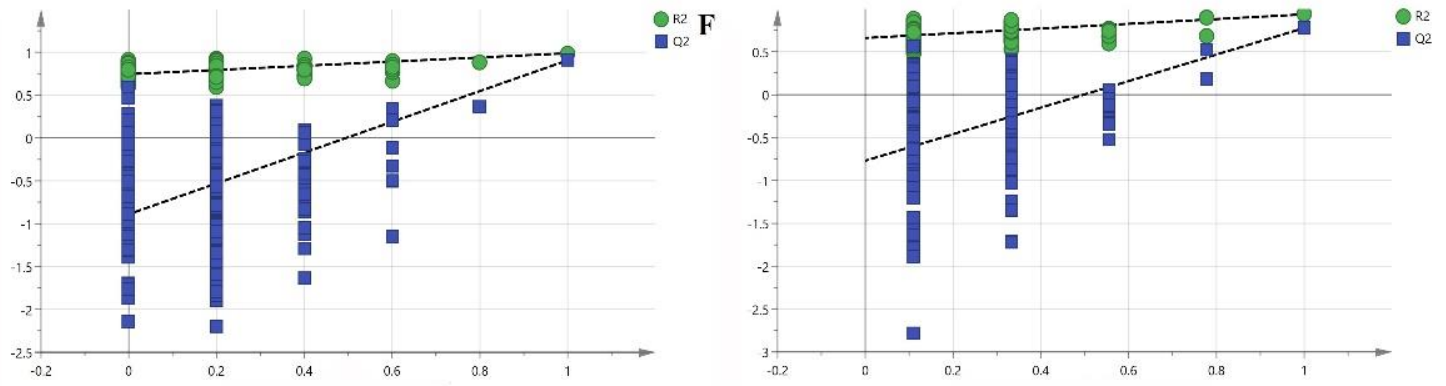

Figure 2. Multivariate statistical analysis of control group, AAI group, one-week group in serum and liver samples. (A) principal components analysis (PCA) scores plot of three groups in serum. (B) PCA scores plot of three groups in liver. (C) orthogonal projections to latent structures discriminant analysis (OPLS-DA) scores scatter plot in serum. (D) OPLS-DA scores scatter plot in liver. (E) OPLS-DA validation plot in serum. (F) OPLS-DA validation plot in liver.

Taken together, as shown in the Venn diagram (Figure 3), compared with the control group, 26 common lipid markers were observed in both serum and liver, which illustrated a close relationship between the hepar and blood in many aspects and the circulating serum lipid profile might reflect the status of the liver function. The data were shown in Table S1 and Table S2. Additionally, in the AAI group, 33 lipids were altered in serum individually (Table S3). Eighteen lipids were identified in liver as well (Table S4). The lipids changed levels among the three groups werein difference, but the tendency of these variations was consistent (Figure 4). For instance, compared with the control group, the level of PE (0:0/18:2) were descended significantly in both serum and liver in AAI group. Then, the level returned obviously in serum, whereas it exhibited the increased tendency with no significance in liver of the one-week group. Compared with control group, the level of PE (0:0/18:0) in AAI group was significantly decreased in both liver and serum samples, and ascended remarkably in the one-week group compared with AAI group. 


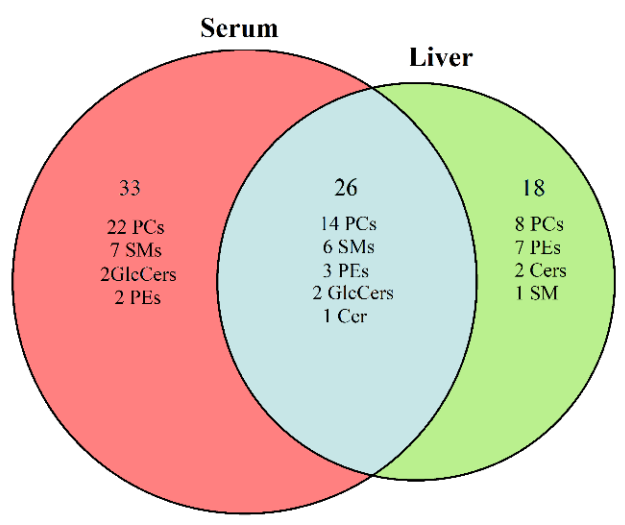

Figure 3. Venn diagram. Each circle represented the potential lipid markers based on the comparisons of the AAI group versus the control group in serum and liver, respectively.

To evaluate the lipid markers' discriminative validity, the receiver operating characteristic (ROC) curve analysis was further performed in the validation set. The results indicated that nine out of 26 identified lipid markers including PC (0:0/19:0), PC (19:0/0:0), SM (d18:2/23:0), SM (d17:1/24:0), SM (d18:1/24:0), SM (d17:1/26:1), SM (d18:1/26:1), GlcCer (d18:1/23:0), and GlcCer (d18:1/24:0) exhibited excellent diagnostic abilities to differentiate between the control group and the AAI group. As shown in Figure 5, nine lipid markers in both liver and serum had the high sensitivity and specificity with the area under the curve (AUC) of 0.740 or larger. Therefore, they might be the suitable metabolites for the detection of AAI hepatotoxicity. Specifically, SM (d18:2/23:0) exhibited the distinguished ability with an AUC value of 0.990 , a specificity of $95.0 \%$ in serum, as well as an AUC value of 0.910 , a specificity of $75.0 \%$ in liver.

Changes in the lipid markers were closely associated with histopathological and biochemical abnormalities of liver. The underlying mechanism responsible for the observed alterations of multiple lipid species in the liver and serum of rats with AAI is presently unclear. Phospholipid metabolism has been proven to be critically associated with the liver regeneration [16]. A number of previous studies have reported the relation between the abnormal phospholipids and the liver failure $[17,18]$. In the lipid metabolism network, phospholipids containing one of the 20:4 fatty acid chain were the reservoir of a series of biologically active lipid mediators. It has been reported that patients with steatohepatitis had less hepatic arachidonic acid (20:4 n-6) than healthy people [19]. In this study, as the main source of arachidonic acid, PCs were converted into it under the inflammatory condition, which caused the metabolic disturbance of inflammatory mediators and the levels of phospholipids were decreased in the AAI group. The previous research also supported the results [20]. In addition, pretreatment with PC could significantly prevent the increases of serum ALT and AST, and reduce the reactive oxygen species levels [21]. This might illustrate that the level of ALT and AST were reversed back to the normal tendency after discontinuance of AAI for two weeks.

SMs were a source of bioactive metabolites to regulate many cellular processes [22]. Deregulation of SM synthesis and transport were associated with a variety of metabolic disorders [23]. Previous studies have been confirmed that SMs and their metabolism played significant roles in regulating the hepatic ischemia, ischemia/reperfusion (I/R) injury, drug-induced injury such as the acetaminophen (APAP) toxicity, and the liver regeneration. They had a great impact on the therapeutic development of diverse liver diseases and might serve as the prognostic and diagnostic markers [24,25]. Cers were mainly synthesized via de novo synthesis and also produced by degradation of sphingomyelin or cerebrosides [26,27]. A series of studies have reported that the levels of LDL, HDL, and VLDL cholesterol in serum exhibited a tendency to decline in patients with liver decompensation [28,29]. SMs and Cers were all involved in VLDL, LDL, and HDL. It was assumed that SMs and Cers lipid species would also be reduced in liver injury $[28,30,31]$. 


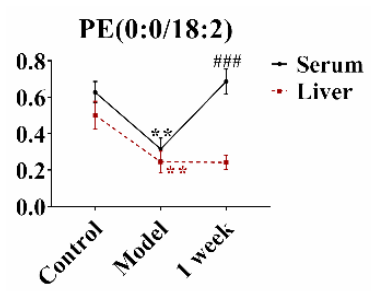

PC(17:0/0:0)

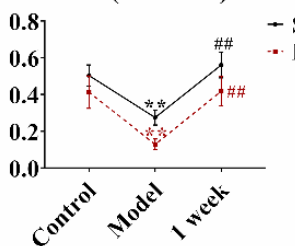

PC(20:5/0:0)

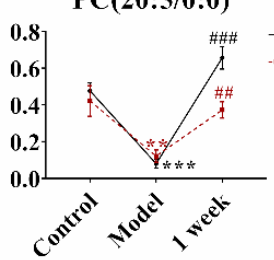

PC(O-16:0/16:1)

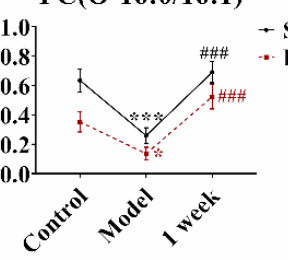

PC(P-18:0/22:6)
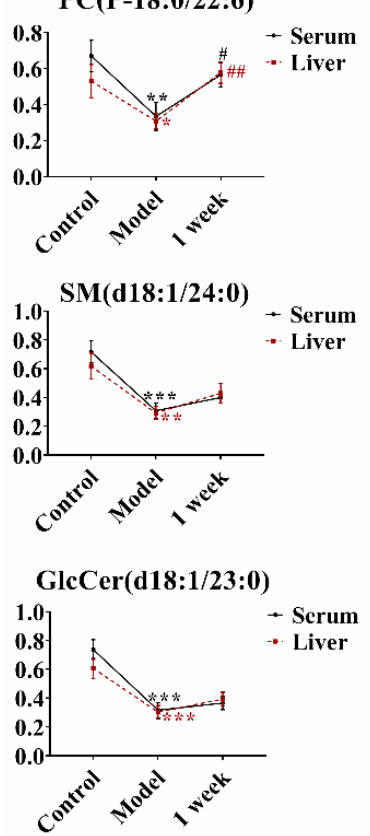

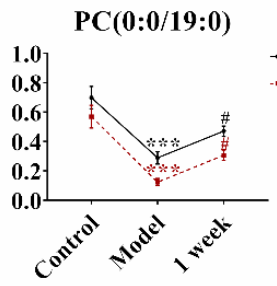

PE(0:0/18:0)

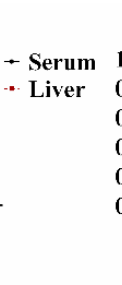

- Serum 0.8
PC(19:0/0:0)

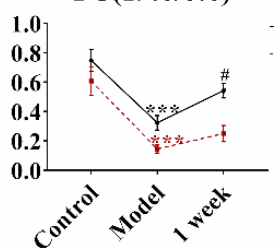

PE(18:0/0:0)

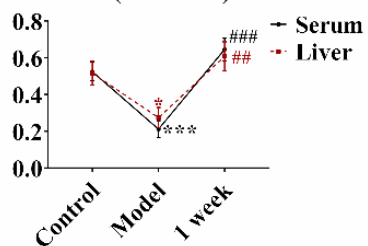

PC(18:2/19:0)

PC(18:0/20:2)
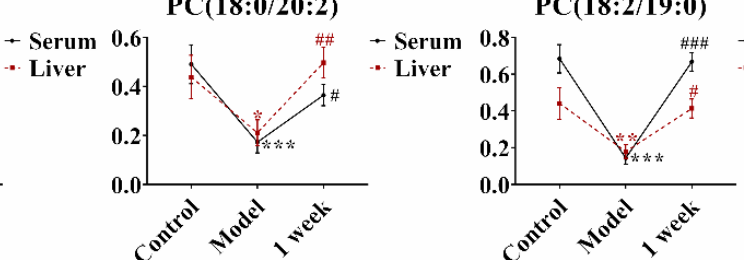

- Serum

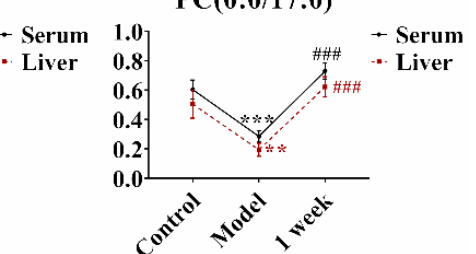

PC(0:0/20:5)

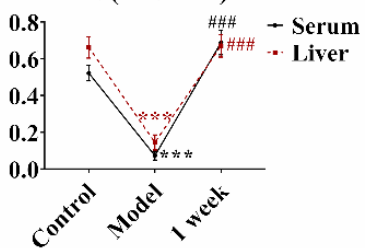

PC(20:4/19:0)

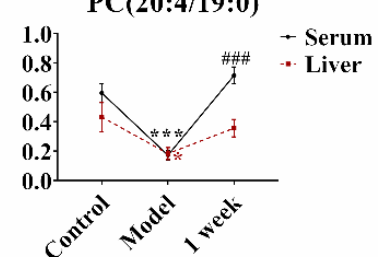

PC(O-18:0/20:4)
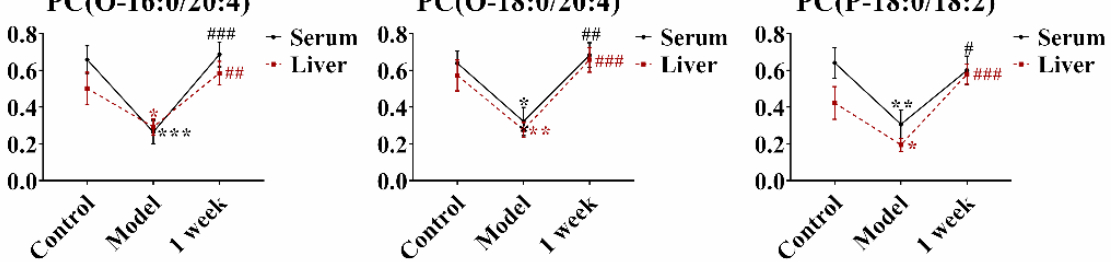

$\operatorname{SM}(d 17: 1 / 24: 0)$

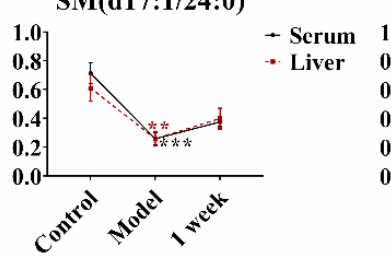

SM(d17:1/24:1)
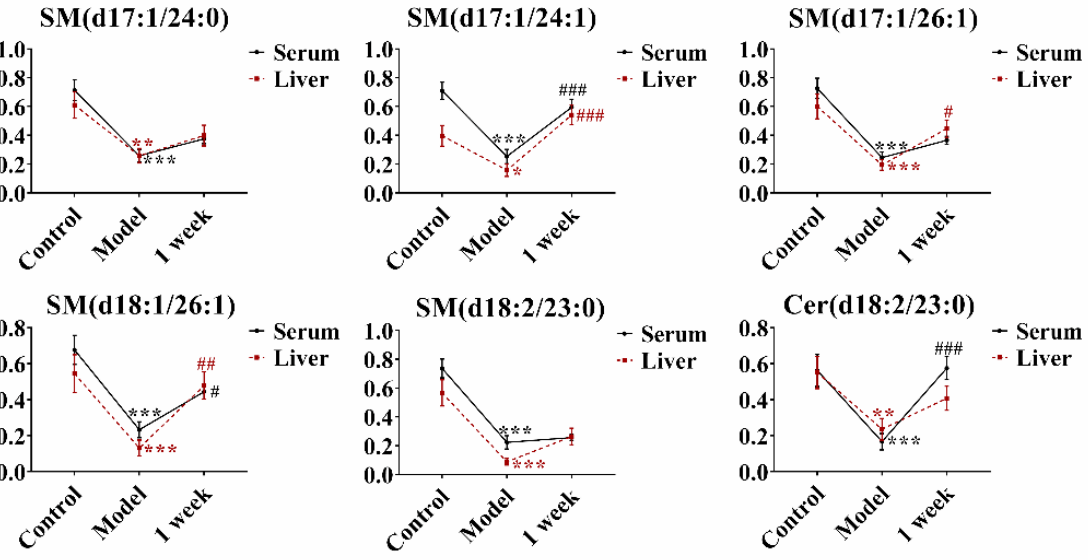

Figure 4. The tendency of level variation of significantly differentially expressed lipids in both liver and serum in the control group, AAI group, and one-week group. ${ }^{*} P<0.05$, ${ }^{* *} P<0.01$, and ${ }^{* * *} P<0.001$, the control group compared to the AAI group. ${ }^{\#} P<0.05$, ${ }^{\# \#} P<0.01$, and ${ }^{\# \# \#} P<0.001$, one-week group compared to the control group. 

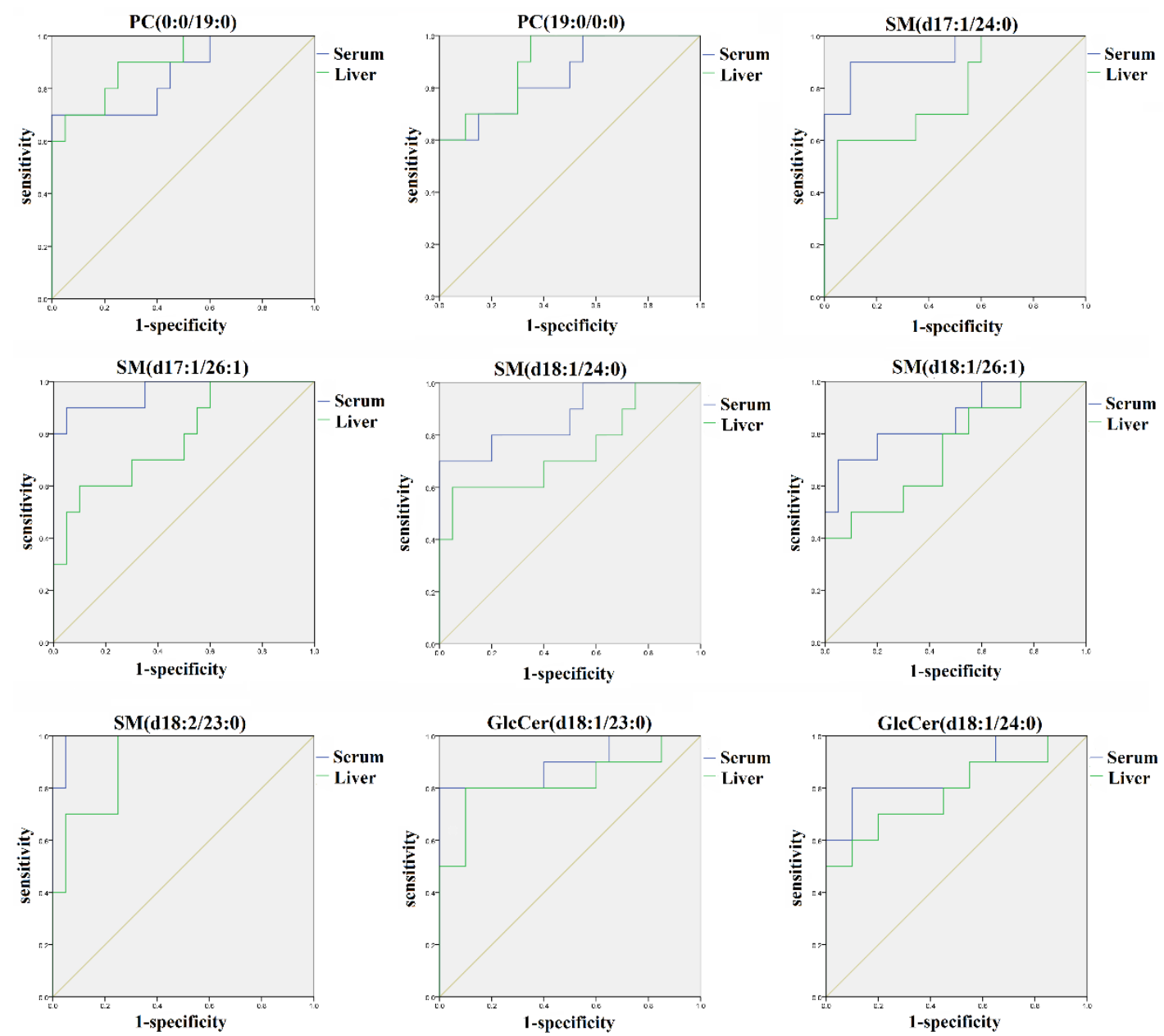

Figure 5. Receiver operating characteristic (ROC) curves of nine out of 26 common lipids from the control group, AAI group, and one-week group based on OPLS-DA analysis. The associated AUC, specificity, and sensitivity values of five SMs, two PCs, and two GlcCers were exhibited.

GlcCers, a type of SMs, were generated by glucosylceramide synthase (GCS). GCS could vary the hepatic lipid metabolism and improve glucose tolerance [32,33]. Mammalian GlcCers mainly contain sphingosine (d18:1). The changes of GlcCer (d18:1/23:0) and GlcCer (d18:1/24:0) were both decreased in the AAI group. The inhibition of GCS was able to trigger the proliferation or apoptosis of hepatic cells and cascadedly lead to the decrease of GlcCer levels. Then, the exerted pro-mitogenic effects happened, which contributed to the process of decreased activity of hepatocytes [34-36]. Additionally, it has been reported that GluCer (d18:1/24:0) could reflect the severity of drug-induced hepatic phospholipidosis in hepatocytes and would be a potential blood biomarker for the diagnosis.

Changes of the lipid markers were closely associated with toxicity. In addition to the hepatotoxicity, PC (18:3/0:0) has been reported to be the suitable metabolic marker with the high sensitivity and specificity for early nephrotoxicity prior to the conventional biochemical or histological abnormalities [37]. Moreover, the plasma lipidomic analysis in humans manifested the relationship of PC species such as PC (38:3), PC (18:2/0:0) et al. with risk measures of metabolic diseases [38-40]. Long chain SMs such as SM (d18:1/24:1) has been found to be downregulated in hepatitis patients compared with in healthy controls [41]. The study demonstrated that the abnormal lipid metabolism was associated with the increased expression of COX-2, ROS, and NF- $\mathrm{kB}$ activation, as well as the inflammatory cell infiltration and fatty degeneration in the chronic kidney disease of mice [42].

In general, the association between the altered lipidomic profilings and AAI hepatotoxicity was explored. The results manifested that the hepatic and serous lipid metabolism had the remarkable correlation with liver function. The perturbations of the lipids homeostasis could be partly reversed 
after the discontinuance of AAI for one week. This was corresponding to the histological evaluation that the hepatic lesion began to be lightened gradually after AAI discontinuance. The identified lipid biomarkers provided a possibility for diagnosing AAI liver injury in an early detection.

\section{Materials and Methods}

\subsection{Chemicals and Reagents}

Aristolochic acid I (AAI) and aristololactam I (ALI) were obtained from Saibaicao Technology Co., Ltd. (Beijing, China). AAI was suspended in $0.1 \%$ sodium carboxymethylcellulose (CMC-Na) solution. Buspirone was purchased from the National Institutes for Food and Drug Control of China (Beijing, China). Methanol and acetonitrile of mass spectrometry (MS) grade were obtained from the Thermo Fisher Company (Fair Lawn, NJ, USA). Formic acid was purchased from Sigma Chemical Co., Ltd. (ST Louis, MO, USA). The purities of all the standard references were over $98 \%$. All other chemicals and solvents were of analytical grade.

\subsection{Animals and Sample Collection}

Male SD rats, weighting $260 \pm 20$ g, were purchased from the Beijing Animals Science Biotechnology Co., Ltd. (Beijing, China). After one week of adaptive feeding, the rats were randomly divided into four groups: Control group, AAI oral-administration group (AAI group), discontinuance of AAI for one-week group (one week group), and discontinuance of AAI for two- week group (two week group), with 10 rats in each group. The AAI group was orally administrated $30 \mathrm{mg} / \mathrm{kg}$ body weight/ day of AAI. All of the hepatic and renal tissues were flash-frozen in liquid nitrogen after washing with normal saline, and were stored at $-80^{\circ} \mathrm{C}$ until analysis. All serum samples were centrifuged at $3000 \mathrm{rpm}, 4{ }^{\circ} \mathrm{C}$ for $10 \mathrm{~min}$ and the supernatant was collected and stored at $-80^{\circ} \mathrm{C}$ freezer until use.

\subsection{Biochemical Analysis}

The levels of serum biochemistry including creatinine (Crea), alanine aminotransferase (ALT), aspartate aminotransferase (AST), blood urea nitrogen (BUN), uric acid (UA), lactic dehydrogenase (LDH), total protein (TP), albumin (ALB), globulin (GLB), total bile acid (TBA), and cholinesterase (CHE) were detected by the Olympus AU480 automated biochemistry analyzer (Olympus, Tokyo, Japan).

\subsection{Histological Evaluation}

Liver and kidney tissues were fixed by $4 \%$ paraformaldehyde solution, dehydrated, embedded in paraffin, prepared at $4 \mu \mathrm{m}$ thickness, and stained with hematoxylin and eosin (H\&E), then observed and described in a panoramic scanner for histopathological examination.

\subsection{Sample Preparation for Lipidomics}

\subsubsection{Treatment of Serum Samples}

The serum samples were thawed at room temperature. $600 \mu \mathrm{L}$ chloroform/methanol (3:1) was added to an aliquot of $100 \mu \mathrm{L}$ serum and sonicated for $3 \mathrm{~min}$. Then, $100 \mu \mathrm{L}$ water was added for vortex-mixing, and centrifuging at $12,000 \mathrm{rpm}, 4^{\circ} \mathrm{C}$ for $10 \mathrm{~min} .300 \mu \mathrm{L}$ of the subnatant chloroform was collected to concentrate under the stream of nitrogen, adding $400 \mu \mathrm{L}$ isopropanol /acetonitrile (1:1) to redissolve, then sonicated and centrifugated at $12,000 \mathrm{rpm}, 4{ }^{\circ} \mathrm{C}$ for $10 \mathrm{~min}$ again. The supernatant was finally analyzed by UPLC-Q-Exactive MS.

\subsubsection{Treatment of Liver Samples}

The liver samples were thawed at room temperature, homogenated, and added $1 \mathrm{~mL}$ chloroform/methanol (3:1), then sonicated for $1 \mathrm{~h}$. The mixture was added $100 \mu \mathrm{L}$ water for vortex-mixing, centrifuging at $13,200 \mathrm{rpm}, 4{ }^{\circ} \mathrm{C}$ for $10 \mathrm{~min}$. Then, $500 \mu \mathrm{L}$ of the subnatant chloroform 
was concentrated under the stream of nitrogen. Adding $300 \mu \mathrm{L}$ isopropanol/acetonitrile (1:1) to the dried samples, vortex-mixing for $40 \mathrm{~s}$ and centrifuging at $12,000 \mathrm{rpm}, 4{ }^{\circ} \mathrm{C}$ for $5 \mathrm{~min}$, finally the supernatant was taken for analysis.

\subsection{Quantitative Analysis of AAI and ALI in Liver and Serum}

\subsubsection{Preparation of Stock Solutions, Calibration Standards, and Internal Standard Solutions}

For the preparation of calibration curves, standard stock solutions contained AAI and ALI were prepared in the methanol at the concentration of $1.0 \mathrm{mg} / \mathrm{mL}$. Working standard solutions were prepared by serial dilution of the stock solutions with the concentrations of 10,000, 8000, 5000, 2000, 1000, $500,200,100$, and $50 \mathrm{ng} / \mathrm{mL}$ to create the necessary concentrations. Buspirone as internal standard (IS) was prepared at $25 \mathrm{ng} / \mathrm{mL}$ in acetonitrile/methanol (1:1). All solutions were stored at $4{ }^{\circ} \mathrm{C}$ for further analysis.

\subsubsection{Preparation for the Serum and Liver Samples}

Aliquots of $50 \mu \mathrm{L}$ serum or homogenated extraction of liver samples were accurately taken and $5 \mu \mathrm{L}$ methanol was added, then spiked with $300 \mu \mathrm{L}$ internal standard solution. After the sufficient vortex for $3 \mathrm{~min}, 4000 \mathrm{rpm}, 3 \mathrm{~min}$ centrifugation was performed at $4{ }^{\circ} \mathrm{C}$ for $15 \mathrm{~min}$. Finally, the supernatant was taken for the LC-MS/MS analysis.

\subsubsection{UPLC-MS/MS Conditions}

Agilent 1200 HPLC (Agilent Technologies, Santa Clara, CA, USA) was used. Kinetex-C 18 110A column $\left(3 \times 30 \mathrm{~mm}, 2.6 \mu \mathrm{m}\right.$, Phenomenex) was operated at $30^{\circ} \mathrm{C}$ and the flow rate was set at $0.8 \mathrm{~mL} / \mathrm{min}$ for separation of AAI and ALI. The mobile phase consisted of (A) water containing of $10 \mathrm{mmol} / \mathrm{L}$ ammonium acetate and (B) acetonitrile. Gradient conditions were as follows: $0-0.5 \mathrm{~min}, 10 \% \mathrm{~B}$; $0.5-0.8 \mathrm{~min}, 10-95 \% \mathrm{~B} ; 0.8-2.5 \mathrm{~min}, 95 \%$ B. A $10 \mu \mathrm{L}$ aliquot of each sample was injected. All samples were kept at $4{ }^{\circ} \mathrm{C}$ throughout the analysis.

MS was performed on an API 4000 Qtrap system (Applied Biosystems, Foster City, CA, USA). Electrospray ionization (ESI) was performed in the positive ion mode. Curtain gas (CUR), nebulizer gas (GS1), and turbo-gas (GS2) were set at 15 psi, $60 \mathrm{psi}$, and 60 psi, respectively. The ionspray voltage was $5.0 \mathrm{kV}$, and the temperature was $550^{\circ} \mathrm{C}$. Nitrogen was employed as the collision gas. AAI and ALI were analyzed using the scheduled MRM. Data acquisitions were performed using the Analyst 1.5.2 software (Applied Biosystems). Multiquant software (Applied Biosystems) was used to quantify AAI and ALI.

\subsubsection{Analytical Validation}

A typical standard curve was prepared by adding the internal standard to serial dilution of the stock solution. Linear regression analysis obtained from the calibration curve was used to calculate the corresponding AAI and ALI in samples. LOQ was determined from the standard solution with the concentration that resulted in a peak with a signal to noise ratio $(\mathrm{S} / \mathrm{N})$ greater than 10:1.

\subsection{UPLC/Q-Exacitve/MS Analysis for Lipidomics}

The UltiMate ${ }^{\mathrm{TM}} 3000$ Rapid Separation LC (RSLC) system (Thermo Scientific, USA) was performed for the relative quantification of various species of lipids. For C18 separation, the binary gradient program consisted of acetonitrile/water (60/40) (mobile phase A) and isopropanol/ acetonitrile (90/10) (mobile phase B). Both A and B contained $0.1 \%$ formic acid and $10 \mathrm{mmol} / \mathrm{L}$ ammonium acetate. All the samples were eluted using the following linear gradient conditions: $0-2 \mathrm{~min}, 20-30 \% \mathrm{~B} ; 2-5 \mathrm{~min}$, $30-45 \%$ B; 5-6.5 min, 45-60\% B; 6.5-12 min, 60-65\% B; 12-14 min, 65-85\% B; 14-17.5 min, 85-100\% $\mathrm{B} ; 17.5-18 \mathrm{~min}, 100-100 \% \mathrm{~B}$, and the equilibration time was $1.5 \mathrm{~min}$ with $20 \% \mathrm{~B}$. The Waters Acquity 
UPLC HSS T3 column $(2.1 \times 100 \mathrm{~mm}, 1.8 \mu \mathrm{m})$ was operated at $50{ }^{\circ} \mathrm{C}$ and the flow rate was set at $0.3 \mathrm{~mL} / \mathrm{min}$ for separation of lipids.

A Thermo Scientific ${ }^{\mathrm{TM}} \mathrm{Q}$ Exactive hybrid quadrupole Orbitrap mass spectrometer equipped with a HESI-II probe was performed in a positive ionization mode. The HESI-II spray voltages was $3.7 \mathrm{kV}$. The heated capillary temperature was $320^{\circ} \mathrm{C}$. The heated vaporizer temperature was $300^{\circ} \mathrm{C}$. The sheath gas pressure was $30 \mathrm{psi}$. The auxiliary gas setting was $10 \mathrm{psi}$ and the collision gas was at a pressure of 1.5 mTorr. The parameters of the full mass scan were resolution of 70,000, auto gain control target under $1.0 \times 106$, maximum isolation time of $50 \mathrm{~ms}$, and the scanning range of $\mathrm{m} / \mathrm{z}$ was 50-1500 Da.

\subsection{Data processing and Statistical Analysis}

All of the LC-MS data were processed by the Progenesis QI software (Nonlinear Dynamics, Newcastle, UK) for peak alignment and peak extraction, providing chromatogram retention time, $\mathrm{m} / \mathrm{z}$, and characteristic peak strength information for further statistical analysis.

Quality control (QC) samples were prepared by mixing equal aliquots of all the samples. The cluster of the QC samples in the PCA scores scatter plot exhibited a satisfactory stability and repeatability of the lipidomic analysis approach. PCA scores plot has been added in Figure S1. In this experiment, five blank samples and following three QC samples were used first to balance the column conditions. Then, one QC sample was inserted every 6-8 samples for monitoring the stability and repeatability of the whole liquid quality system. The multivariate analysis was performed by SIMCA14.1 (Umetrics AB, Umea, Sweden) including principal component analysis (PCA) and orthogonal partial least squares discriminant analysis (OPLS-DA). Based on the criteria of variable importance in the projection (VIP) values (VIP $>1$ ) obtained from the OPLS-DA model, t-test $(P<0.05)$ and fold change values $(\mathrm{FC}>1.5$ or FC $<0.7$ ) was set as the indexes for screening the potential biomarkers with significant difference. Then, using the Skyline software for the relative quantification of the selected potential lipid structures through multistage mass spectra information fragments. In the receiver operating characteristic (ROC) curve analysis, the diagnostic index of sensitivity and specificity of lipids in serum and liver tissue was calculated by the SPSS 22.0 software (IBM Crop., Armonk, NY, USA). Youden index = sensitivity ( 1 - specificity). The maximum of Youden index was defined as the cutoff value of the lipid metabolism.

All data were presented as means \pm standard error of the mean (SEM). Statistical significance between the different groups was determined by one-way ANOVA followed by least significant difference (LSD) post hoc tests using GraphPad prism version 7.01 (GraphPad Software, Inc. Version 7.01). Statistically significant level was set at $P<0.05$.

\section{Conclusions}

In the present study, the kidney was the main impaired target organ for AA-induced injury compared with liver damage. Along with the AAI discontinuance, the lesion severity was alleviated. The concentrations of AAI and ALI in liver and serum could not be detected in the discontinuance groups according to the LC-MS/MS quantitative analysis. A total of 44 lipid markers in liver, as well as 59 in serum were identified. Twenty-six common lipids were observed in both serum and liver. According to the evaluation of histological characteristics, serum biochemical indexes, and the lipidomic profilings, it indicated that the hepatotoxicity induced by AAI could be reversible to some extent. Furthermore, the hypothesis that the hepatocyte damage would be related to the liver cancer needs further research in the future.

Supplementary Materials: The following are available online. Table S1: Identification of 26 common lipid markers in serum of AAI group and control group; Table S2: Identification of 26 common lipid markers in liver of AAI group and control group; Table S3: Identification of 33 lipid markers in serum of AAI group and control group; Table S4: Identification of 18 lipid markers in liver of AAI group and control group; Figure S1: (A) The PCA scores plot of QC and on-test samples in serum. (B) The PCA scores plot of QC and on-test samples in liver.

Author Contributions: J.Z. performed the experiments and wrote the original draft. Y.Y. analyzed the data of the histopathological section. H.W. assisted in data analyses. B.B., J.Y., X.W., and Y.Z. discussed the results and 
commented on the manuscript. N.S. and H.Z. contributed to the study design and reviewed and edited the manuscript. All the authors reviewed the manuscript.

Funding: This research was funded by the National Science and Technology Major Project (2015ZX09501004-003-001 and 2018ZX09101002-003), China Academy of Chinese Medical Sciences Foundation (ZZ10-025 and ZZ12-001), and the Major International S\&T Cooperation Project from Ministry of Science and Technology of the People's Republic of China (No. 2016YFE0129000).

Conflicts of Interest: The authors declare no conflict of interest.

\section{References}

1. Li, J.; Zhang, L.; Jiang, Z.; Shu, B.; Li, F.; Bao, Q.; Zhang, L. Toxicities of aristolochic acid I and aristololactam I in cultured renal epithelial cells. Toxicol. In Vitro 2010, 24, 1092-1097. [CrossRef] [PubMed]

2. Jiang, Z.Z.; Bao, Q.L.; Sun, L.X.; Huang, X.; Wang, T.; Zhang, S.; Li, H.; Zhang, L.Y. Possible role of mtDNA depletion and respiratory chain defects in aristolochic acid I-induced acute nephrotoxicity. Toxicol. Appl. Pharmacol. 2013, 266, 198-203. [CrossRef] [PubMed]

3. Zhu, S.; Wang, Y.; Jin, J.; Guan, C.; Li, M.; Xi, C.; Ouyang, Z.; Chen, M.; Qiu, Y.; Huang, M.; et al. Endoplasmic reticulum stress mediates aristolochic acid I-induced apoptosis in human renal proximal tubular epithelial cells. Toxicol. In Vitro 2012, 26, 663-671. [CrossRef] [PubMed]

4. $\quad$ Li, Y.C.; Tsai, S.H.; Chen, S.M.; Chang, Y.M.; Huang, T.C.; Huang, Y.P.; Chang, C.T.; Lee, J.A. Aristolochic acid-induced accumulation of methylglyoxal and nepsilon-(carboxymethyl) lysine: An important and novel pathway in the pathogenic mechanism for aristolochic acid nephropathy. Biochem. Biophys. Res. Commun. 2012, 423, 832-837. [CrossRef] [PubMed]

5. Stiborová, M.; Arlt, V.M.; Schmeiser, H.H. DNA Adducts Formed by Aristolochic Acid Are Unique Biomarkers of Exposure and Explain the Initiation Phase of Upper Urothelial Cancer. Int. J. Mol. Sci. 2017, 18, 2144. [CrossRef]

6. Lu, Z.N.; Luo, Q.; Zhao, L.N.; Shi, Y.; Wang, N.; Wang, L.; Han, Z.G. Mutational Features of Aristolochic Acid-Induced Mouse and Human Liver Cancers. Hepatology 2019. [CrossRef]

7. Rolim, A.E.; Henrique-Araújo, R.; Ferraz, E.G.; de Araújo Alves Dultra, F.K.; Fernandez, L.G. Lipidomics in the study of lipid metabolism: Current perspectives in the omic sciences. Gene 2015, 554, 131-139. [CrossRef]

8. Griffiths, W.J.; Ogundare, M.; Williams, C.M.; Wang, Y. On the future of "omics": Lipidomics. J. Inherit. Metab. Dis. 2011, 34, 583-592. [CrossRef]

9. Han, X. Lipidomics: Developments and applications. J. Chromatogr. B. Analyt. Technol. Biomed. Life Sci. 2009, 877, 2663. [CrossRef]

10. Wu, C.; Zhang, Y.; Chai, L.; Wang, H. Histological changes, lipid metabolism and oxidative stress in the liver of, Bufo gargarizans, exposed to cadmium concentrations. Chemosphere 2017, 179, 337-346. [CrossRef]

11. Khattab, H.; Fouad, A.; Hamza, M.; Mohey, M.A.; El-Akel, W.; Ghoneim, H.; Abul-Fotouh, A.; Esmat, G. Relation of ALT and AST levels to the histopathological changes in liver biopsies of patients with chronic hepatitis C genotype 4. Arab. J. Gastroenterol. 2015, 16, 50-53. [CrossRef] [PubMed]

12. Angulo, P.; Keach, J.C.; Batts, K.P.; Lindor, K.D. Independent predictors of liver fibrosis in patients with nonalcoholic steatohepatitis. Hepatology 1999, 30, 1356-1362. [CrossRef] [PubMed]

13. Park, G.; Lin, B.P.; Ngu, M.C.; Jones, D.B.; Katelaris, P.H. Aspartate aminotransferase: Alanine aminotransferase ratio in chronic hepatitis $\mathrm{C}$ infection: Is it a useful predictor of cirrhosis? J. Gastroenterol. Hepatol. 2000, 15, 386-390. [CrossRef] [PubMed]

14. Zhang, G.S. The clinical application value of biochemical test in the diagnosis of patients with cirrhosis. Guide China Med. 2016, 14, 148.

15. Yang, Q.H. The clinical application value analysis of biochemical test in the diagnosis of liver cirrhosis. JACM 2018, 11, 47-48.

16. Park, E.S.; Lee, J.H.; Hong, J.H.; Park, Y.K.; Lee, J.W.; Lee, W.J.; Lee, J.W.; Kim, K.P.; Kim, K.H. Phosphatidylcholine Alteration Identified Using MALDI Imaging MS in HBV-Infected Mouse Livers and Virus-Mediated Regeneration Defects. PLoS ONE 2014, 9, e103955. [CrossRef]

17. Li, Z.; Guan, M.; Lin, Y.; Cui, X.; Zhang, Y.; Zhao, Z.; Zhu, J. Aberrant Lipid Metabolism in Hepatocellular Carcinoma Revealed by Liver Lipidomics. Int. J. Mol. Sci. 2017, 18, 2550. [CrossRef] 
18. Ming, Y.N.; Zhang, J.Y.; Wang, X.L.; Li, C.M.; Ma, S.C.; Wang, Z.Y.; Liu, X.L.; Li, X.B.; Mao, Y.M. Liquid chromatography mass spectrometry-based profiling of phosphatidylcholine and phosphatidylethanolamine in the plasma and liver of acetaminophen-induced liver injured mice. Lipids Health Dis. 2017, 16, 153. [CrossRef]

19. Puri, P.; Baillie, R.A.; Wiest, M.M.; Mirshahi, F.; Choudhury, J.; Cheung, O.; Sargeant, C.; Contos, M.J.; Sanyal, A.J. A lipidomic analysis of nonalcoholic fatty liver disease. Hepatology 2007, 46, 1081-1090. [CrossRef]

20. Zhao, L.L. UPLC-Q/TOF MS Analysis of GP Metabolism in RAW264.7 Cell Inflammation Model Interfering by NSAIDs; GDPU: Guangzhou, China, 2012.

21. Na, J.Y.; Song, K.; Kim, S.; Kwon, J. Hepatoprotective effect of phosphatidylcholine against carbon tetrachloride liver damage in mice. Biochem. Biophys. Res. Commun. 2015, 460, 308-313. [CrossRef]

22. Hannun, Y.A.; Obeid, L.M. Sphingolipids and their metabolism in physiology and disease. Nat. Rev. Mol. Cell Biol. 2018, 19, 175-191. [CrossRef] [PubMed]

23. Régnier, M.; Polizzi, A.; Guillou, H.; Loiseau, N. Sphingolipid metabolism in non-alcoholic fatty liver diseases. Biochimie 2019, 159, 9-22. [CrossRef] [PubMed]

24. Nojima, H.; Freeman, C.M.; Gulbins, E.; Lentsch, A.B. Sphingolipids in liver injury, repair and regeneration. Biol. Chem. 2015, 396, 633-643. [CrossRef] [PubMed]

25. Li, J.F.; Qu, F.; Zheng, S.J.; Ren, J.Y.; Wu, H.L.; Liu, M.; Liu, H.; Ren, F.; Chen, Y.; Zhang, J.L.; et al. Plasma sphingolipids as potential indicators of hepatic necroinflammation in patients with chronic hepatitis $\mathrm{C}$ and normal alanine aminotransferase level. PLoS ONE 2014, 9, e95095. [CrossRef] [PubMed]

26. Bartke, N.; Hannun, Y.A. Bioactive sphingolipids: Metabolism and function. J. Lipid Res. 2009, 50, S91-S96. [CrossRef] [PubMed]

27. Lahiri, S.; Futerman, A.H. The metabolism and function of sphingolipids and glycosphingolipids. Cell Mol. Life Sci. 2007, 64, 2270-2284. [CrossRef] [PubMed]

28. Breier, C.; Lisch, H.J.; Braunsteiner, H. Lipoproteins, HDL-apolipoproteins, activities of hepatic lipase and lecithin-cholesterol acyltransferase in the plasma of patients with post-alcoholic end-stage liver cirrhosis. Klin. Wochenschr. 1983, 61, 929-931. [CrossRef]

29. Vere, C.C.; Streba, C.T.; Streba, L.; Rogoveanu, I. Rogoveanu, Lipid serum profile in patients with viral liver cirrhosis. Med. Princ. Pract. 2012, 21, 566-568. [CrossRef]

30. Krautbauer, S.; Eisinger, K.; Wiest, R.; Liebisch, G.; Buechler, C. Systemic saturated lysophosphatidylcholine is associated with hepatic function in patients with liver cirrhosis. Prostaglandins Other Lipid Mediat. 2016, 124, 27-33. [CrossRef]

31. Krautbauer, S.; Wiest, R.; Liebisch, G.; Buechler, C. Associations of systemic sphingolipids with measures of hepatic function in liver cirrhosis are related to cholesterol. Prostaglandins Other Lipid Mediat. 2017, 131, 25-32. [CrossRef]

32. Margalit, M.; Shalev, Z.; Pappo, O.; Sklair-Levy, M.; Alper, R.; Gomori, M.; Engelhardt, D.; Rabbani, E.; Ilan, Y. Glucocerebroside ameliorates the metabolic syndrome in OB/OB mice. J. Pharmacol. Exp. Ther. 2006, 319, 105-110. [CrossRef] [PubMed]

33. Zigmond, E.; Zangen, S.W.; Pappo, O.; Sklair-Levy, M.; Lalazar, G.; Zolotaryova, L.; Raz, I.; Ilan, Y. $\beta$-Glycosphingolipids improve glucose intolerance and hepatic steatosis of the Cohen diabetic rat. Am. J. Physiol. Endocrinol. Metab. 2009, 296, E72-E78. [CrossRef] [PubMed]

34. Li, J.F.; Zheng, S.J.; Wang, L.L.; Liu, S.; Ren, F.; Chen, Y.; Bai, L.; Liu, M.; Duan, Z.P. Glucosylceramide synthase regulates the proliferation and apoptosis of liver cells in vitro by Bcl-2/Bax pathway. Mol. Med. Rep. 2017, 16, 7355-7360. [CrossRef] [PubMed]

35. Datta, S.C.; Radin, N.S. Stimulation of liver growth and DNA synthesis by glucosylceramide. Lipids 1988, 23, 508-510. [CrossRef] [PubMed]

36. Marsh, N.L.; Elias, P.M.; Holleran, W.M. Glucosylceramides stimulate murine epidermal hyperproliferation. J. Clin. Investig. 1995, 95, 2903-2909. [CrossRef]

37. Zhao, Y.Y.; Wang, H.L.; Cheng, X.L.; Wei, F.; Bai, X.; Lin, R.C.; Vaziri, N.D. Metabolomics analysis reveals the association between lipid abnormalities and oxidative stress, inflammation, fibrosis, and Nrf2 dysfunction in aristolochic acid-induced nephropathy. Sci Rep. 2015, 5, 12936. [CrossRef]

38. Kulkarni,H.; Meikle, P.J.; Mamtani, M.; Weir, J.M.; Barlow, C.K.; Jowett, J.B.; Bellis, C.; Dyer, T.D.; Johnson, M.P.; Rainwater, D.L.; et al. Variability in associations of phosphatidylcholine molecular species with metabolic syndrome in Mexican-American families. Lipids 2013, 48, 497-503. [CrossRef] 
39. Syme, C.; Czajkowski, S.; Shin, J.; Abrahamowicz, M.; Leonard, G.; Perron, M.; Richer, L.; Veillette, S.; Gaudet, D.; Strug, L.; et al. Glycerophosphocholine metabolites and cardiovascular disease risk factors in adolescents: A cohort study. Circulation 2016, 134, 1629-1636. [CrossRef]

40. Zeng, H.; Tong, R.; Tong, W.; Yang, Q.; Qiu, M.; Xiong, A.; Sun, S.; Ding, L.; Zhang, H.; Yang, L. Metabolic biomarkers for prognostic prediction of pre-diabetes: Results from a longitudinal cohort study. Sci. Rep. 2017, 7, 6575. [CrossRef]

41. Chen, S.L.; Yin, P.Y.; Zhao, X.J.; Xing, W.B.; Hu, C.X.; Zhou, L.N.; Xu, G.W. Serum lipid profiling of patients with chronic hepatitis B, cirrhosis, and hepatocellular carcinoma by ultra fast LC/IT-TOF MS. Electrophoresis 2013, 34, 2848-2856. [CrossRef]

42. Gui, S.X.; Li, B.Y.; Zhao, X.Y.; Sheng, L.; Hong, J.; Yu, X.H.; Sang, X.Z.; Sun, Q.Q.; Ze, Y.G.; Wang, L.; et al. Renal Injury and Nrf2 Modulation in Mouse Kidney Following Chronic Exposure to TiO2 Nanoparticles. J. Agric. Food Chem. 2013, 61, 8959. [CrossRef] [PubMed]

(C) 2019 by the authors. Licensee MDPI, Basel, Switzerland. This article is an open access article distributed under the terms and conditions of the Creative Commons Attribution (CC BY) license (http://creativecommons.org/licenses/by/4.0/). 